\title{
A BAYESIAN BELIEF NETWORK METHOD FOR BRIDGE DETERIORATION DETECTION
}

\author{
Matteo Vagnoli', Rasa Remenyte-Prescott ${ }^{1 *}$, John Andrews ${ }^{1}$ \\ ${ }^{1}$ Resilience Engineering Research Group, University of Nottingham \\ Science Road, University Park, \\ NG7 2RD, Nottingham, United Kingdom
}

*Corresponding author: r.remenyte-prescott@nottingham.ac.uk

\begin{abstract}
Bridges are one of the most important assets of transportation networks. A closure of a bridge can increase the vulnerability of the geographic area served by such networks, as it reduces the number of available routes. Condition monitoring and deterioration detection methods can be used to monitor the health state of a bridge and enable detection of early signs of deterioration. In this paper, a novel Bayesian Belief Network (BBN) methodology for bridge deterioration detection is proposed. A method to build a BBN structure and to define the Conditional Probability Tables (CPTs) is presented first. Then evidence of the bridge behaviour (such as bridge displacement or acceleration due to traffic) is used as an input to the BBN model, the probability of the health state of whole bridge and its elements is updated and the levels of deterioration are detected. The methodology is illustrated using a Finite Element Model (FEM) of a steel truss bridge, and for an in-field post-tensioned concrete bridge.
\end{abstract}

Keywords: Bayesian Belief Network, bridge deterioration, detection and diagnostics, structural health monitoring. 


\section{Introduction}

The European transportation network has more than one million of bridges [European Commission, 2012]. These assets are continuously deteriorating due to traffic loading and environmental effects. Time-consuming and expensive visual inspection techniques are widely adopted to assess the health state of bridges ranging from one to six years [Moughty and Casas, 2017]. Detailed visual examinations of the bridge critical elements require interruption of service, and the knowledge about the location of the deteriorated bridge element [Fan and Qiao, 2011]. Conversely, Structural Health Monitoring (SHM) methods are used to assess the health state of bridges, remotely and continuously, by relying on the analysis of static and dynamic responses of bridges [Rice et al., 2011]. Therefore, SHM methods can support bridge owners to detect ongoing degradation promptly, and optimize the maintenance schedule accordingly, by minimizing the whole life cycle cost of the asset [Frangopol et al., 2012].

In recent years, SHM and condition monitoring methods have been developed widely to monitor and evaluate the health state of bridge elements. Particularly, model-based and non-model-based methods have been introduced. The former methods assess the health state of a bridge, by comparing the behaviour of the in-field bridge with a Finite Element Model (FEM) of the bridge; the latter methods assess the health state of the bridge by analysing the measured behaviour of the in-field bridge directly. In this respect, several SHM methods for condition monitoring and damage detection of bridges are presented in literature, as reviewed in [Fan and Qiao, 2011; Moughty and Casas, 2017; Vagnoli et al., 2018], with several challenges to be addressed. For example, model-based methods, such as FEM updating methods [Sanayei et al., 2015], require a complex and time-consuming procedure to develop a reliable FEM. Therefore, continuous condition monitoring might not be achieved. Model-based methods can detect damage existence and its location, but they show difficulties in diagnosing the causes [Vagnoli et al., 2018]. In contrast, non-model-based methods, such as Artificial Neural Networks [Arangio and Beck, 2012], Principal Component Analysis [Hsu and Loh, 2010], supervised and unsupervised clustering techniques [Alves et al., 2015], show promising results for continuous condition monitoring of bridges. However, the performance of non-model-based methods strongly depends on the quality of available data. For instance, when modal parameters of the bridge are used as an input to a non-model-based SHM method, false alarms and misleading results can be obtained, due to the noise in data which affects a reliable extraction of the bridge modal parameters [Moughty and Casas, 2017]. In fact, low frequency modal parameters of the bridge, which can be extracted from the measured 
data reliably, are of low sensitivity to damage [Kim et al., 2015]. Conversely, high frequency modal parameters of the bridge, which are more sensitive to damage, are difficult to extract from the measured data in a reliable manner [Casas and Moughty, 2017]. Furthermore, non-model-based methods usually do not consider the knowledge of structural engineers that design and maintain bridges, and the influence of degradation of individual elements on the health state of the whole bridge. Finally, it is worth mentioning that both model-based and non-model-based methods need a supply of reliable data about the behaviour of the structure, in order to monitor the condition of the structure [Lédeczi et al., 2009]. Necessary data come from a measurement system, which can be based on: i) a traditional approach with one type of sensors, such as strain gauges, accelerometers, tiltmeters, vision systems, optometers, fibre optic, piezoelectric sensors or GPS; or ii) a hybrid and integrated sensors system, consisting of multiple types of sensors (i.e. fiber optics, GPS, accelerometers, etc.). The measurement system is chosen by bridge manager based on the requirements of the monitoring: indeed, the health state of the bridge can be monitored with different level of accuracy by different type of sensors, e.g. accelerometers allow to monitor the health state of a bridge accurately, but they have been recently outperformed by fiber optics in terms of accuracy [Psimoulis and Stiros, 2013; Bao et al., 2017; Vagnoli et al., 2018]. In this paper, a Bayesian Belief Network (BBN) method for deterioration detection of bridges is presented. Although BBN methods are well known in literature, they are commonly used for reliability assessment of bridges [Holický et al., 2013; Franchin et al., 2016; Martínez-Martínez et al., 2017]. In addition, Bayesian approaches have been used to assess the health state of bridges [Mustafa and Matsumoto, 2017; Ni et al., 2019; Zheng and Yu, 2015]. However, these approaches mainly focus on updating the estimation of the bridge materials property (e.g. the values of the bridge stiffness) and behaviour (e.g. updating the mode shapes), and consequently, the influence of each bridge element on the health state of the whole bridge is not assessed.

Therefore, a BBN-based approach is proposed in this paper, which is used for evaluating and updating the bridge health state. This approach is based on the analysis of data provided by sensors installed on the bridge, and it is also based on the assessment of the influence of each bridge element on the health state of the bridge. Indeed, the proposed BBN method allows to update the health state of the whole bridge and its elements, by taking account of the health state of bridge elements. Furthermore, different sources of information can be used in combination as inputs to the BBN model, such as (continuous) sensor data and state evaluations from visual inspections of the bridge. The BBN model 
is developed by identifying major (such as the deck, the chords, etc.) and minor (such as the diagonals, verticals, etc.) elements of the bridge, represented by a node. Nodes are connected from minor to major elements. The Conditional Probability Tables (CPTs) are built by adopting a Fuzzy Analytic Hierarchy Process (FAHP) of bridge expert judgements [Loughney and Wang, 2017]. The model is then used to update the health state of the whole bridge and its elements, by introducing the evidence about bridge behaviour into the model. First of all, this process is illustrated for a steel truss bridge, which is modelled using a FEM model, representing effects of different levels of degradation to the behaviour of the bridge. The displacement of the bridge joints is modelled to simulate the formation and propagation of microcracks at the joint location(s) [Mehrjoo et al., 2008]. Secondly, the method is also illustrated for an infield post-tensioned concrete bridge. Bridge acceleration data for a number of different states of degradation are used when the bridge is excited by environmental factors (such as wind). Overall, the proposed BBN method is used to detect the time when the health state of the bridge degrades, by diagnosing the location and magnitude of the minor element(s) of the bridge that are affecting the health state of the bridge. Some good performance of the method is illustrated in both case studies.

The paper is organised as follows: the steps to build a BBN model are given in Section 2; Section 3 presents the application of the proposed method in the two case studies; conclusions and future challenges are discussed in Section 4.

\section{The proposed Bayesian Belief Network method for bridge degradation detection}

The theoretical background of the BBN is out of scope of this paper, and an interested reader can find details in [Jensen and Nilsen, 2007]. Briefly, a BBN consists of a structure that is formed of a set of variables (called nodes), and a set of directed links (called arcs) between system variables of interest. In addition to its structure, BBNs contain a quantitative part, which is represented using Conditional Probability Tables (CPTs) associated with each node. In this paper, conditional probabilistic relationships between connected nodes of the BBN are described by discrete conditional probability distributions, as proposed by [Morales-Nápoles et al., 2014]. This is due to the fact that the health state of a bridge is usually described by discrete states, for example, good, degraded and failed condition, [Rafiq et al., 2015].

\subsection{Overview of the proposed methodology}

A BBN approach for bridge deterioration detection is developed by following the procedure, presented in Figure 1. Firstly, a BBN model for a bridge is built using bridge information (Section 2.2); then the 
CPTs can be defined through an expert knowledge elicitation process (Section 2.3) [Loughney and Wang, 2017]. Once the BBN model is developed, the condition of the bridge can be evaluated. Information about bridge behaviour, such as data provided by sensors installed on the bridge or visual inspection reports, as well as modelling results of deterioration scenarios, can be used as an input to the BBN model. The levels of deterioration of the bridge and its elements are obtained using the BBN approach, after the evidence about bridge behaviour is introduced in the model and the updated probabilities of being in each state are calculated.

It is intended, that such an approach can be used by a bridge manager to monitor the evolution of the health state of the bridge over time, and detect which elements are degrading, so that appropriate maintenance actions can be initiated. Hence, Section 2.2 shows a detailed step-by-step description of how to define and build the BBN structure to represent the bridge structure. Similarly, Section 2.3 presents a detailed description of the process for the CPTs definition, by showing how a bridge manager can develop CPTs using the information obtained from bridge expert questionnaires. Finally, Section 3 shows two case studies of the proposed method, in order to illustrate how to adopt the BBN method for monitoring the health state of a bridge.

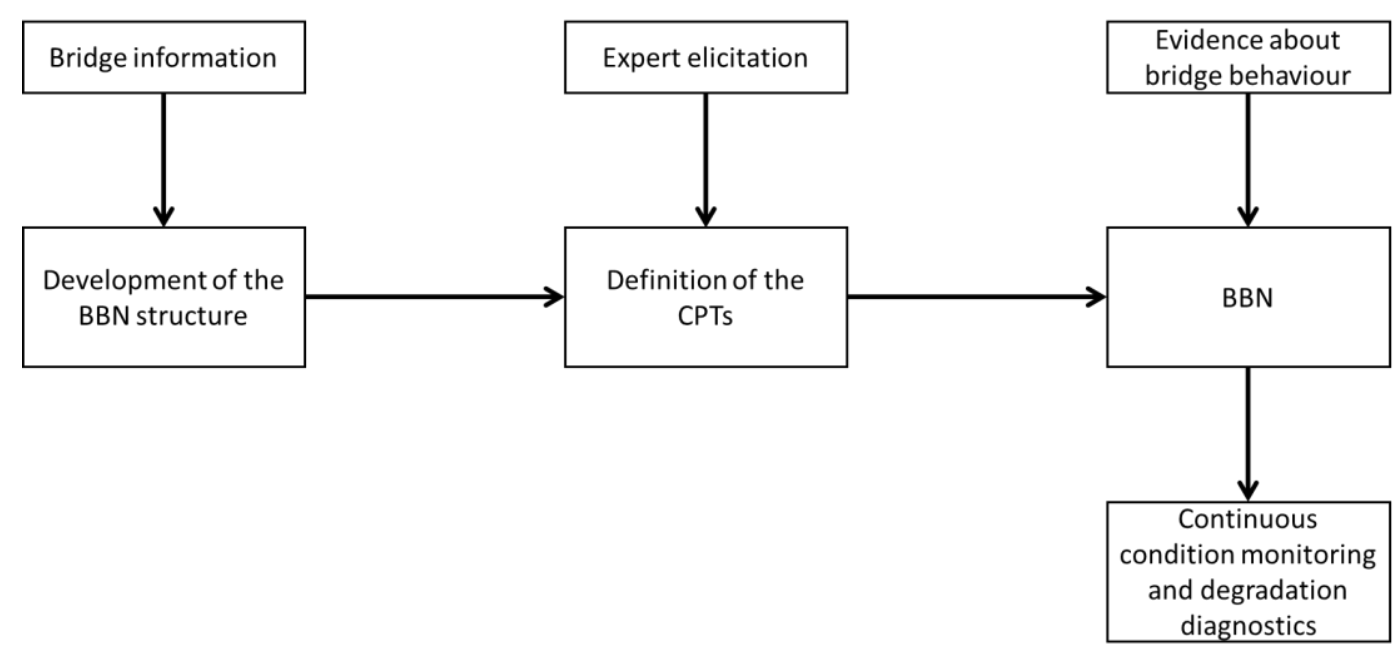

Figure 1. A BBN method for bridge degradation detection

\subsection{Building the BBN model}

A step-by-step process to develop a BBN model for a bridge is hereafter described. The following steps are proposed in order to develop the structure of the BBN:

1. Identify the type of the bridge and its major and minor elements. The identification and analysis of the bridge structure, such as a truss, arch or suspension [Gentile and Saisi, 2015]), 
helps to define its major and minor elements. The level of detail depends on the requirements of a bridge manager. For example, each minor element is made of relatively small elements, such as smaller beams and joints, therefore, further detail beyond minor elements (e.g., bearings, bridge anchors, rivets, etc) can be included. The size of the BBN depends on the identification of the major and minor elements of interest, and thus major and minor elements should be selected by guaranteeing a manageable size of the BBN [Rafiq et al., 2015]. Indeed, if an analysis beyond the minor elements is needed to assess the health state of each bridge component (e.g. rivets, bearing, small beams, etc.), the size of the traditional BBN can increase significantly. As a consequence, the bridge manager would need to consider developing an Object-Oriented BBN (OOBN), in order to have a manageable BBN structure for each minor element of the bridge. In that case the health state of a minor element would be assessed by evaluating the influence between the different OOBNs, each representing the detailed analysis of the minor elements of the bridge.

2. Define the BBN structure. After the bridge elements are identified, the structure of the BBN is developed. The nodes of the BBN represent the major and minor elements, whereas the arcs represent the interdependencies between the elements. The arcs are drawn, assuming that the health state of a major element of the bridge is influenced by the condition of its minor elements. In addition, the health state of a major (minor) element also depends on the health state of other neighbouring major (minor) elements, so more arcs are added, as shown in section 3.1.3 and 3.2.3. As a consequence, the nodes representing the minor elements are called parent nodes, whereas those representing the major elements are called child nodes. This is due to the fact that they are influenced by the health state of their parents (i.e. minor elements). Finally, the health state of the whole bridge is represented by a child node to the bridge major elements [Attoh-Okine and Bowers, 2006].

3. Describe the health states for each node. An element of the bridge goes through a number of states over time, from a healthy to a degraded state and also to a severely degraded condition. Therefore, each BBN node is described by a set of mutually exclusive discrete states, usually well known by bridge owners. For example, Network Rail, which is the owner of the UK railway network, evaluates the bridge condition by three states: i) good condition (maintenance actions are not required); ii) partially degraded (maintenance actions are required, but they can 
be postponed, without compromising the safety of the asset); iii) severely degraded, (essential maintenance actions, i.e. that cannot be postponed, are required) [Rafiq et al., 2015].

4. Add BBN nodes of evidence for a sensor system on the bridge. If a sensor system is installed on the bridge, the data provided by the sensors can be used as evidence of the bridge behaviour. Hence, a node for each sensor is added to the BBN. A sensor node is a parent node of the bridge element(s) where the sensor is installed, and the states of the sensor nodes are defined based on the monitored bridge behaviour. For example, when the bridge vibration is measured by the measurement system of the bridge, the states of the sensor nodes are defined by considering that, given a defined and constant external excitation to the bridge, the higher the vibration of the bridge, the more degraded the bridge. However, if there are no sensors on the bridge, nodes of evidence are added to represent other sources of information, such as visual inspection reports. The nodes of evidence can also represent bridge behaviour, obtained from a FEM model. In this way, the bridge manager can use the available evidence of the bridge behaviour as an input to the BBN nodes of evidence, and thus update the health state of the whole bridge and its elements.

After the BBN structure is completed, the CPTs need to be defined in order to describe the relationships between the nodes in the BBN.

\subsection{Developing CPTs}

CPTs are used to define the dependencies between the nodes of the BBN using conditional probabilities. The strategy of defining the CPTs depends on the nature of the available information: $i$ ) if a database of information about the past behaviour of the system is available, the CPTs can be defined by adopting a learning technique, e.g. expectation maximization [Sun et al., 2006]; ii) if such a database is unavailable, the CPTs can be defined by using an expert knowledge elicitation process [Loughney and Wang, 2017], also used in this paper:

a) Identify the experts. The accuracy of the elicitation process strongly depends on the knowledge of experts. Selecting several experts with different levels of expertise can lead to a more complex analysis of their answers. However, a result that is retrieved by aggregating the analysis from a heterogenic group of experts is usually more reliable than each individual analysis [Kabir et al., 2016]. 
b) Define the degradation scenarios. The uniformity and consistency of the elicitation process can be improved by providing the expert with a small set of highly informative and consistent questions [Elmasry et al., 2017]. For instance, if an expert is asked to assess the degradation of a minor element, the major element, which contains that minor element, is also expected to be in a similar health state [Rafiq et al., 2015].

c) Present the scenarios to the experts. The set of scenarios is given to the experts using interviews and online surveys. Each scenario needs to be described accurately, by explaining the health state of the element and its influence on the health state of the elements, represented by the connected nodes.

d) Provide a scale for answers. A linguistic scale for answering the questions is needed [Torfi et al., 2010], an example is shown in Table 1. In fact, experts can be more comfortable in providing a linguistic answer rather than numerical values of the probability, and thus a linguistic scale can be arbitrarily defined depending on the case study of interest, in order to have some clear and concise response options for the questionnaire.

\begin{tabular}{|c|c|}
\hline $\begin{array}{c}\text { Lingustic } \\
\text { scale }\end{array}$ & Meaning \\
\hline very unlikely & The described degradation scenario is highly unlikely \\
\hline unlikely & The described degradation scenario is unlikely but possible \\
\hline even chance & The described degradation scenario might happen or not \\
\hline likely & The described degradation scenario is likely \\
\hline very likely & The described degradation scenario is highly likely \\
\hline
\end{tabular}

Table 1. Linguistic scale for assessing the interdependencies between bridge elements

e) Merge the individual analyses. The linguistic answers from the experts needs to be converted to a numerical description, using a fuzzy membership function, as shown in Figure 2. In this way, the vagueness and subjectivity of the expert judgment is addressed mathematically, by allowing the user to quantify the answers of the experts (see Section 3.1.4 for a detailed example). The individual membership functions are merged together, by weighing the experience of the experts. In this paper, a weighing factor $\left(W_{1}\right)$ is used to weigh the expert analysis with respect to the level of experience [Kabir et al., 2016]: 


$$
W_{I}=\frac{E_{I}^{\beta}}{\max _{i=1}^{N}\left(E_{i}^{\beta}\right)}
$$

where $E_{l}$ is the number of years of experience of the expert $l ; \beta$ is a parameter employed to adequately weigh the analysis of each expert, and needs to be optimized to guarantee a group judgment [Vagnoli et al., 2017].

f) Assess the influence of each parent node on its child. The influence of a parent node on its child nodes is evaluated by using a Fuzzy Analytics Analytic Hierarchy Process (FAHP) of the experts' analyses. In what follows, only the main output of the FAHP is described; an interested reader can find further details in [Wang and Elhag, 2006; Loughney and Wang, 2017]. The FAHP aims to assess the importance weight vector $\left(w_{h}\right)$ of each parent node on its child nodes, i.e. the influence of each parent node on the health state of its child node:

$$
w_{h}=\frac{I_{h}}{\sum_{h=1}^{D} I_{h}}
$$

where $I_{h}$ is an integral value that evaluates the influence of a parent node on each of its $D$ child nodes [Wang and Elhag, 2006]. $w_{h}$ is then used to define the CPTs.

g) Assess the consistency of the expert analysis. The consistency of the expert analysis needs to be verified by assessing a Consistency Ratio $(C R)$. Therefore, the Consistency Index $(C l)$ is computed as follows:

$$
C l=\frac{\lambda_{\max }-D}{D-1}
$$

where $\lambda_{\max }$ is the maximum eigenvalue of the defuzzified pairwise comparison matrix, i.e. the matrix that groups the merged experts judgment about the influence between different bridge elements. $C R$ is evaluated by dividing $C /$ by a Random Index $(R I)$, which is provided in literature and depends on the size $D$ of the pairwise comparison matrix:

$$
C R=\frac{C l}{R l}
$$

Generally, the FAHP is considered consistent, if the $C R$ is lower than 0.1 [Dağdeviren and Yüksel, 2008]. 


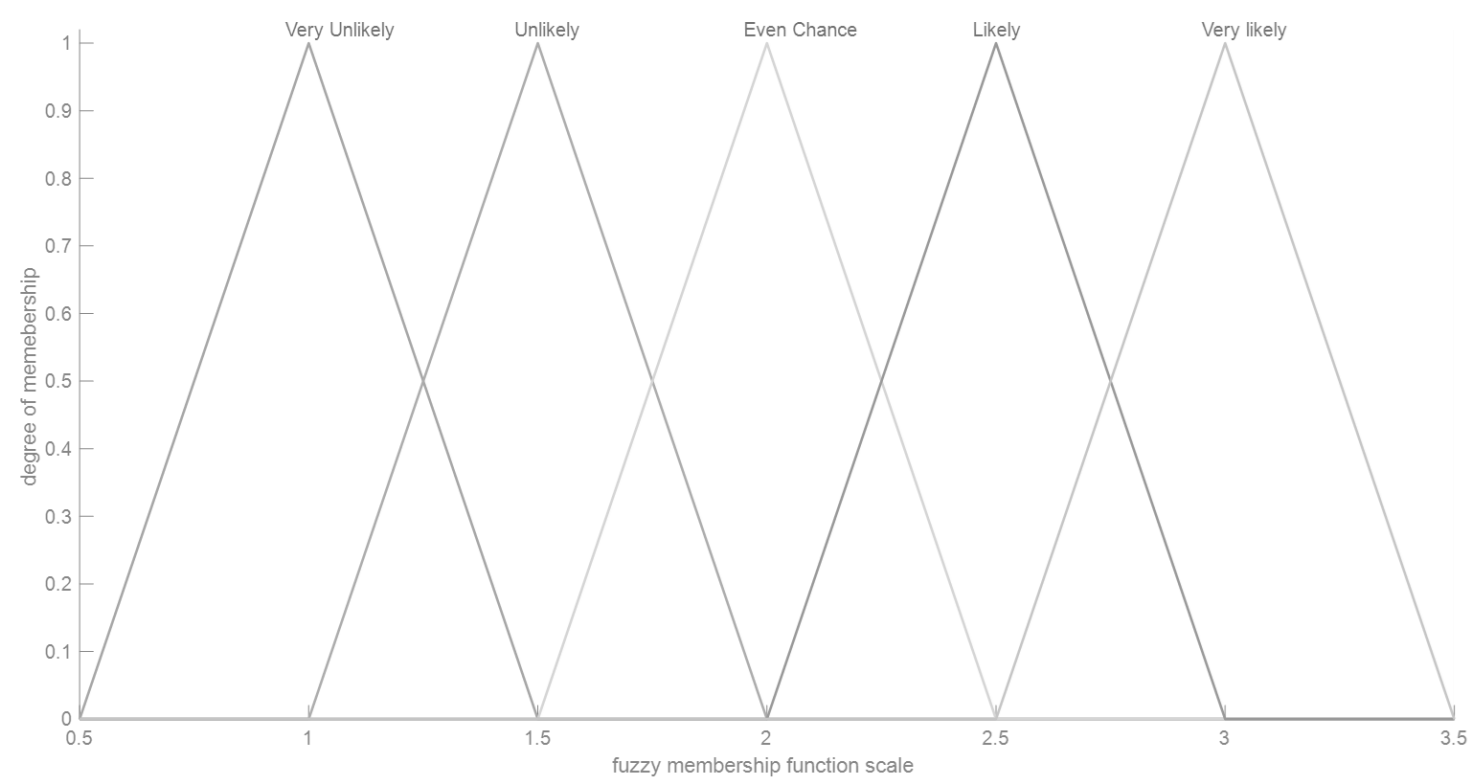

Figure 2. Triangular fuzzy membership function to represent the linguistic scale.

h) Compute the interdependencies between bridge elements. The CPTs are hereafter defined by considering that the degradation of a bridge can be modelled by adopting a linear model [Kreislova and Geiplova, 2012; Attema et al., 2017; Rao et al., 2017]. Assume that a bridge element, described by node $X$, is in state $x_{i}$. There are $N$ parent nodes of this element, denoted as a set $Y^{k}$. The conditional probability is calculated using a linear function shown in Eq. (5):

$P\left(x_{i} \mid \bigcap_{k=1}^{N} Y^{k}=y_{k}\right)=P\left(x_{i} \mid \bigcap_{k=1}^{N} Y^{k}=y_{k}=1\right)+\sum_{m=1}^{M} P\left(x_{i} \mid \bigcap_{k=1}^{N} Y^{k}=y_{k}=1\right) \cdot w_{h_{m}} \cdot f_{p_{m}} \cdot p_{i} \cdot \delta$

where

- $\quad P\left(x_{i} \mid \bigcap_{k=1}^{N} Y^{k}=y_{k}\right)$ is the probability of the child node in state $x_{i}$, with a condition that the health states of the parent nodes are known, denoted as $y_{k}$;

- $\quad P\left(x_{i} \mid \bigcap_{k=1}^{N} Y^{k}=y_{k}=1\right)$ is the probability of the child node $x$ in state $x_{i}$, with a condition that all parent nodes are in the healthy state, $y_{k}=1$, i.e. the parent nodes show no degradation;

- $M$ is the number of degraded parent nodes;

- $w_{h_{m}}$ is the importance weight vector used to assess the influence of each degraded parent node on the state of the child node, note that $w_{h_{m}}$ is calculated using Eq. (2); 
- $f_{p_{m}}$ is a penalty factor that increases as the condition of the bridge element(s) deteriorates; $\delta=-1$ if the child node is in the healthy state, $x_{i}=1$, and $\delta=1$ if the child node is in the degraded state;

- $\quad p_{i}$ is a vector used to normalise the respective column of probabilities in the CPT and it depends on the state of the child node.

Overall, Eq. (5) shows that the probability of the child node being in state $x_{i}$, with a condition that $M$ parent nodes are degraded, is computed as the total $\sum_{m=1}^{M} w_{h_{m}} f_{p_{m}}$ decrease or increase of $P\left(x_{i} \mid \bigcap_{k=1}^{N} Y^{k}=y_{k}=1\right)$, caused by the influence of $M$ parent nodes that are degraded. $M$ can be between 0 to $N$, i.e. if $M=0$ (there are no degraded parent nodes), then $P\left(x_{i} \mid \bigcap_{k=1}^{N} Y^{k}=y_{k}=1\right)$ does not change; if $M=N$ (all parent nodes are degraded), then $P\left(x_{i} \mid \bigcap_{k=1}^{N} Y^{k}=y_{k}=1\right)$ experiences its maximum variation.

Finally, after the CPTs are defined, the BBN model can be used to detect bridge deterioration.

\section{Application of the BBN method to detection of bridge degradation}

The proposed BBN method is illustrated using two case studies, in order to analyse the performance of the BBN method in detecting and bridge deterioration and diagnosing causes of the change in behaviour and the bridge health state:

1. In Section 3.1, an FEM model of a steel truss bridge is used to simulate a large number of scenarios with bridge degradation, results of which are used as inputs to the BBN.

2. In Section 3.2, vibration data of an in-field post-tensioned concrete bridge, which is subjected to a progressive damage test, is used as an input to the BBN.

The following sections show two detailed applications of the proposed method. First the method is applied using an FEM model to represent bridge response due to deterioration. Then, the method is applied to a post-tensioned in-field bridge, and it is illustrated how the evolution of the health state of an in-field bridge can be monitored by a bridge manager, relying on bridge acceleration data. 


\subsection{Application to a steel truss bridge, using an FEM model}

\subsubsection{The FEM of a steel truss bridge}

A warren steel truss bridge is modelled using SAP2000, as shown in Figure 3(a). A steel truss bridge is selected since the degradation mechanisms of steel, such as corrosion and cracks, can develop rapidly after they have been initiated. Hence, an early detection and management of such degraded conditions can improve the safety and availability of the bridge [Ahmadi and Anvari, 2018]. Figure 3(b) shows the top chords of the bridge, which are $24 \mathrm{~m}$ long. Figure 3(c) illustrates the side view of the bridge, where the diagonals elements are $8.55 \mathrm{~m}$ long. Figure $3(\mathrm{~d})$ shows the bridge bottom chords, which are composed of 5 beams. The elements of the bridge are modelled by considering grade S355 steel, as this steel is commonly used in Europe to build steel railway bridges [Vagnoli et al., 2017]. The reference system, depicted in Figure 3, is defined as follows: the right-hand side of the bridge is considered as the side of the bridge at $y=0 \mathrm{~m}$, the left-hand side is considered to be at $y=7 \mathrm{~m}$.

Displacements of the bridge elements at the joints are chosen as the variable to describe the performance of the bridge under load. In fact, displacements of steel bridge elements are a good indicator of the bridge condition, e.g. allowing to detect fatigue damage, and thus to ensure that the steel bridge can be effectively maintained. However, it is worth noting that, despite the recent technology advancement with GPS sensors, cameras and image recognition algorithms, the in-field monitoring of displacements can be challenging [Hester et al., 2017]. The displacement values are obtained at 5 locations on each top chord and 6 locations on each bottom chord, as shown by dark circles in Figure 3. Gaussian noise is added to the simulated displacements, as noisy data is unavoidable in in-field applications [Dowling et al., 2012]:

$$
y_{p o l}=y_{F E}+q_{p} N \sigma\left(y_{F E}\right)
$$

where $y_{p o l}$ is the displacement of a bridge element when the noise is added, $y_{F E}$ is the displacement value provided by the FEM, $q_{p}$ is the ratio of standard deviation between the noise and the FEM displacement $y_{F E}$ and is equal to $5 \%$ [Attema et al., 2017]. $N$ is a standard normal distribution of mean 0 and standard deviation 1 , and $\sigma\left(y_{F E}\right)$ is the standard deviation of the displacement of the FEM elements when the same health state of the bridge is simulated by modifying environmental factors, such as the load on the bridge. 
(a)

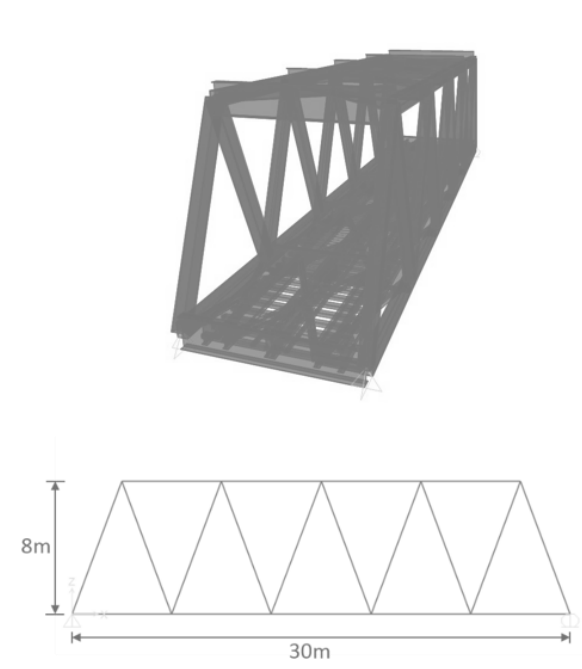

(c) (b)
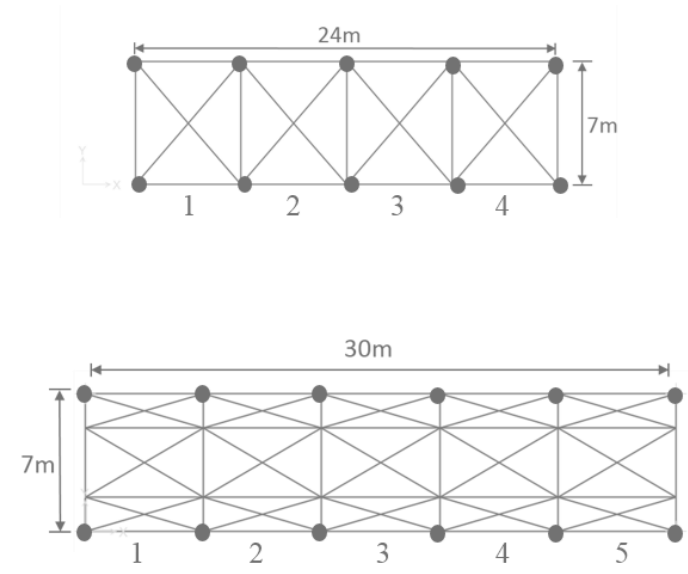

(d)

Figure 3. The FEM model of the steel truss bridge: overview, top, lateral and bottom view, in (a), (b), (c) and (d), respectively.

\subsubsection{Degradation scenarios}

A degradation mechanism of the bridge element(s), considered in this case study, is the formation and propagation of micro-cracks at the joint location. Indeed, micro-cracks are difficult to spot during a visual inspection, and more than $40 \%$ of steel truss bridges are affected by the formation of micro-cracks at the joint location [Mehrjoo et al., 2008]. Bridge displacement is chosen as the variable to represent bridge behaviour. This choice is made due to the limitations of the vibration-based parameters, such as natural frequency and mode shapes, that are usually monitored for SHM purposes [Moughty and Casas, 2017]. However, it should also be noted that some measurement methods for bridge displacement, such as displacement sensors, GPS, strain sensors and laser measurements, can have limitations in terms of precision [Zhao et al., 2015]; bridge displacement is chosen for illustration purposes. In the paper, the deterioration of the bridge element(s) is studied using 28 deterioration scenarios, including individual and multiple elements:

- 22 individual element scenarios describe situations where each joint of the top chords (the first 10 scenarios) and bottom chords (the remaining 12 scenarios) is degraded, by simulating a loss of $30 \%$ of the joint cross-sectional area. The loss of 30\% of cross-section area is selected by considering the average of values for cross-sectional area reduction, studied in a similar truss bridge in [Mehrjoo et al., 2008]. 
- 6 multiple element scenarios include situations, where two or three joints of the top/bottom chords are degraded.

\subsubsection{Building the BBN model for the steel truss bridge}

The development of the structure of the BBN is carried out by following the four steps proposed in Section 2.2:

1. In this case study, the major elements are the top and bottom chords, and the minor elements are all individual beams that belong to a major element. In fact, these elements are usually subjected to the highest stress [Ni et al., 2012].

2. Since each top chord is made of 4 beams, as shown in Figure $3(b)$, therefore, 4 nodes are used in the BBN model to represent these four elements. For example, each $E_{-} j+T C R$ node in Figure 4 represents all minor elements $j$, where $j=1,2,3,4$, of the top chord on the right-hand side of the bridge (TCR) (note that they are also parent nodes of TCR node in Figure 4). In the same way, elements on the left-hand side of the top chord, and on the right and left-hand side of the bottom chord are defined, $E_{-} j_{-} T C L, E \_{ }_{-} B C R$ and $E_{-} j_{-} B C L$, respectively. Finally, we assume that the health state of the bridge (or the deck), represented by the BridgeHealthState node in Figure 4 , is affected by these major elements.

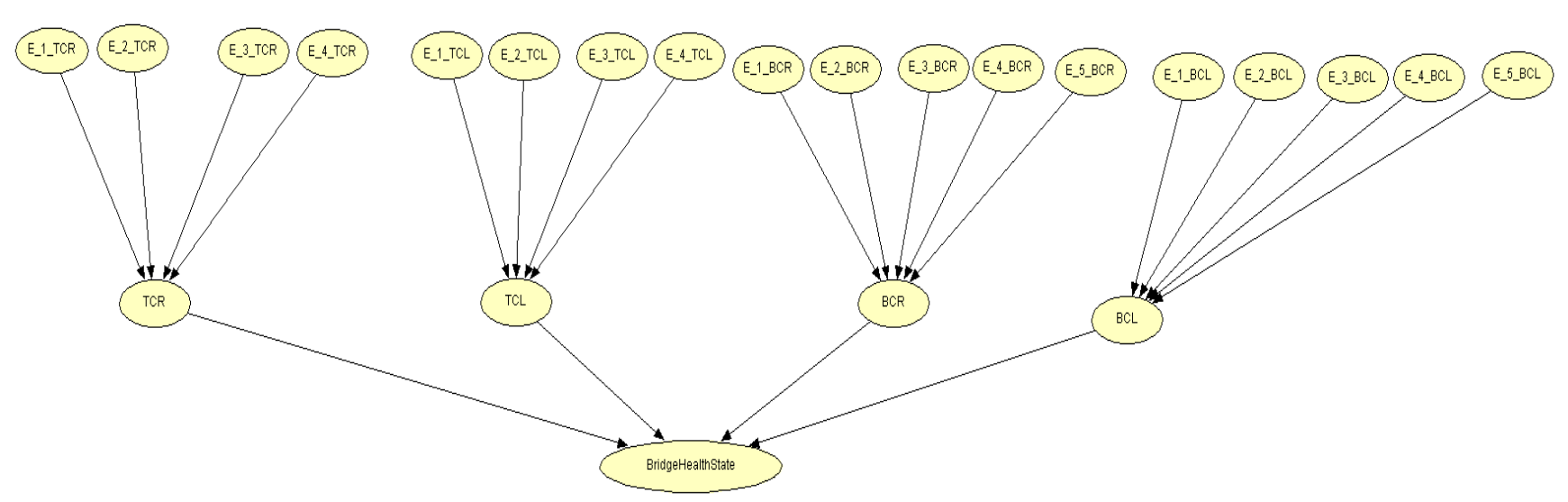

Figure 4. The BBN model for the example steel truss bridge.

In addition, interdependencies among neighbouring major/minor elements are included and more nodes are added in the model. Examples are the nodes ending with "_1" in Figure 5. The nodes Top_chords and Bottom_chords are used in order to group the major elements. 


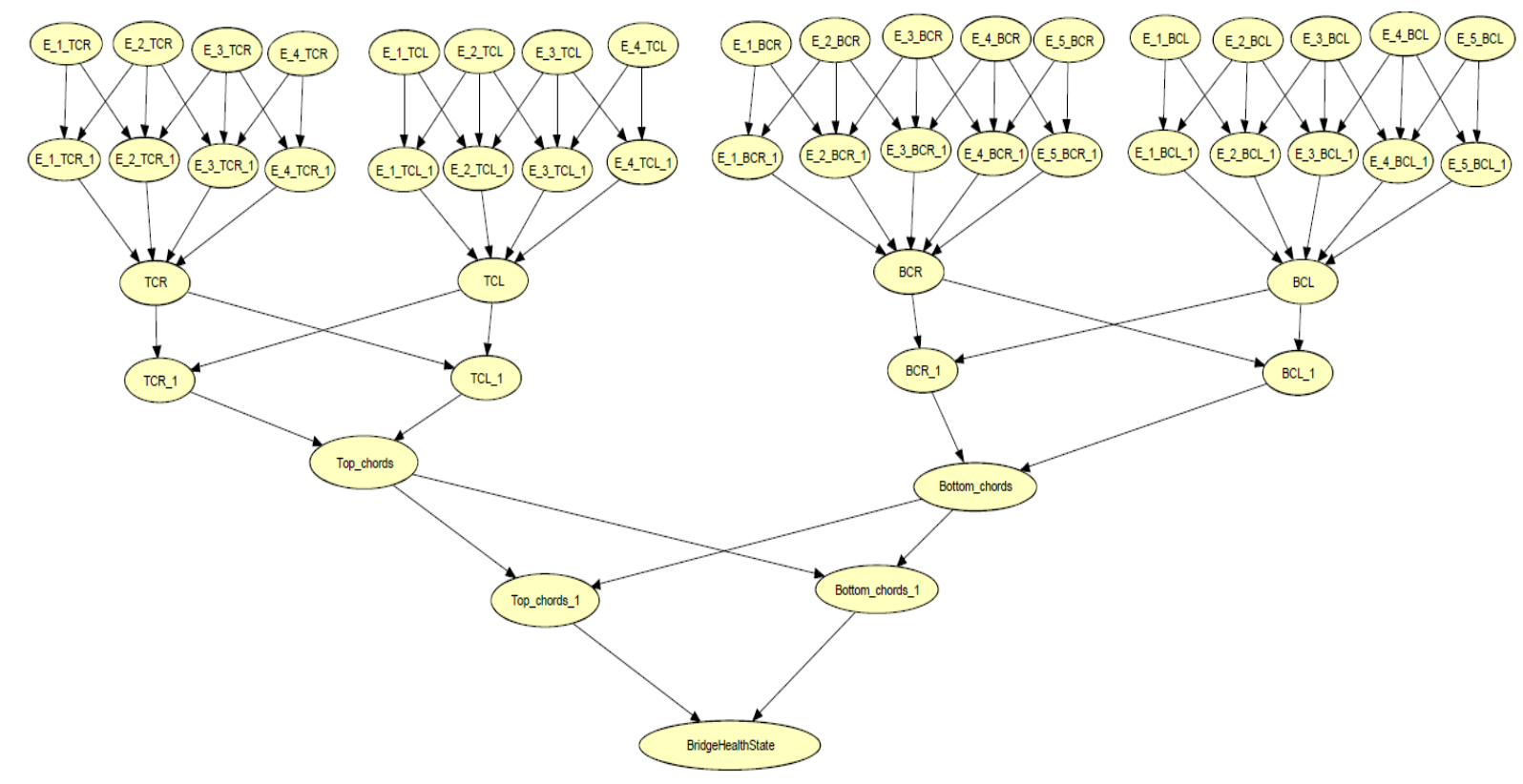

Figure 5 The BBN model with additional interdependencies.

3. As proposed in Section 2.2, three states are considered. These are: a) a healthy state, denoted as $\mathrm{H}$, where the element is in a good condition; $b$ ) a partially degraded state, denoted as PD, where the element can require a maintenance action, which can be postponed; $c$ ) a severely degraded state, denoted as SD, where the element needs to be maintained urgently.

4. Since in this case study no sensors were installed on the bridge, nodes of evidence are added which represent virtual displacement sensors at the joint locations, as shown by dark circles in Figure 3. 5 sensor nodes are related to each top chord and 6 - to each bottom chord. For example, TCR_J_i, where $i=1,2,3,4$, and 5 , represents the sensors on the right-hand side top chord, as shown in Figure 6 . The states of the sensor nodes are related to the amplitude of the bridge behaviour, i.e. the displacement in this case study. For example, given a defined load on the bridge, the higher the displacements of the bridge element, the more degraded the bridge element. 


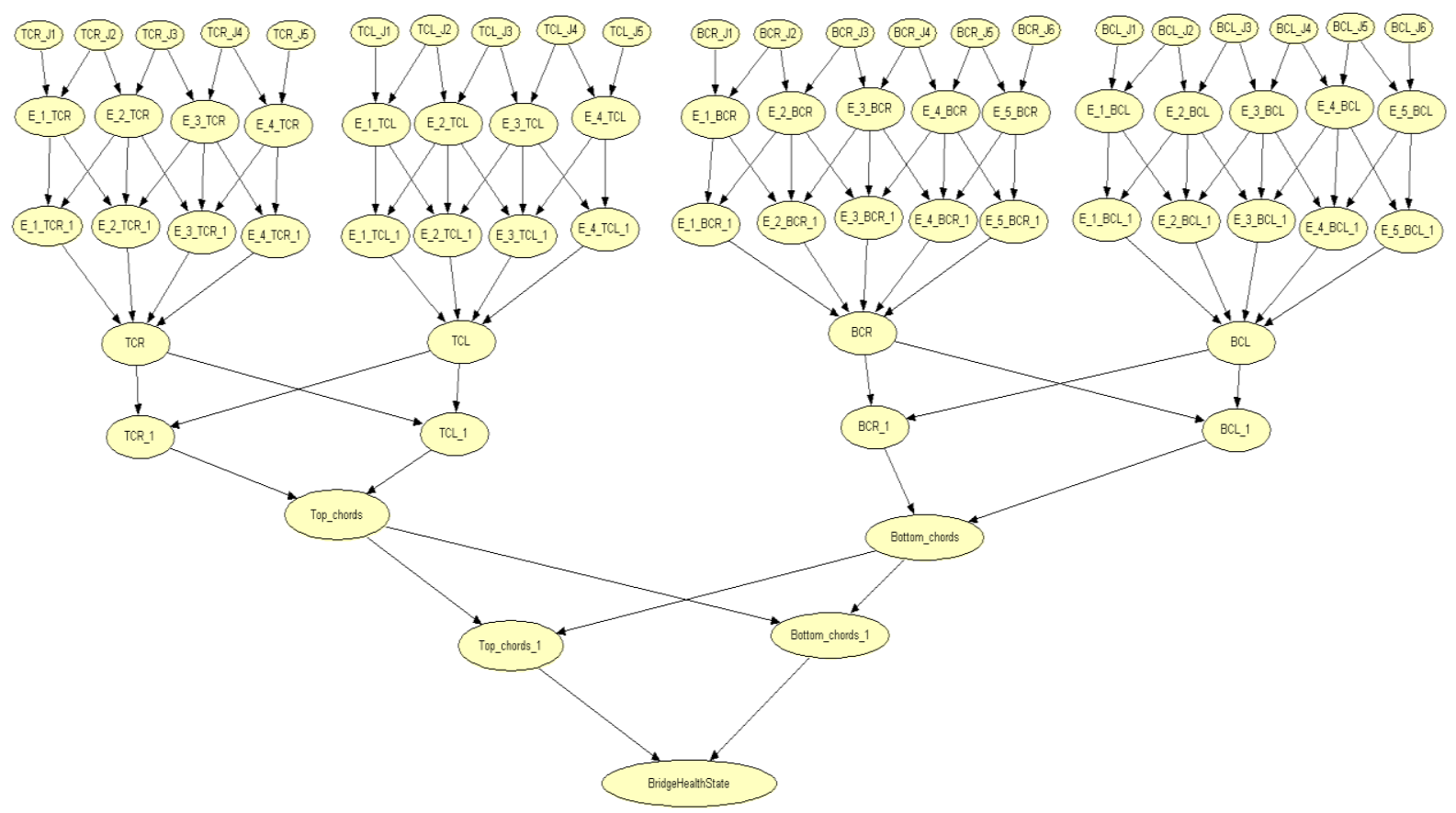

Figure 6. The final BBN model.

\subsubsection{Developing CPTs for the steel truss bridge}

The CPTs are developed by adopting the expert knowledge elicitation process, as described in Section 2.3:

a) Three bridge experts were interviewed: i) Expert 1 is a principal engineer of an engineering firm with 8 years of experience in SHM; ii) Expert 2 is a director of an engineering consultancy group with over 28 years of experience in structural assessment of civil infrastructure; iii) Expert 3 is a professor of structural engineering in a top ranked UK university, with more than 25 years of experience in SHM.

b) The experts were interviewed to evaluate four degradation scenarios: $i)$ the influence of the degradation of a minor element on the health state of a neighbouring minor element; ii) the influence of the degradation of a minor element on the health state of a major element; iii) the influence of the degradation of a major element on the health state of a different major element; iv) the influence of the degradation of a major element on the health state of the whole bridge.

c) The four scenarios were presented to the experts. Without loss of generality, the degradation scenario iii) is hereafter presented. The influence of the top chord on the right-hand side on the health state of other major elements can be analysed by the means of three questions: Let us consider a truss steel bridge, given in Figure 3. 
1. Referring to your expertise, if the top chord on the right-hand side requires essential maintenance, which cannot be postponed, how likely would the health state of the bottom chord on the right-hand side be influenced by this degraded state of the top chord on the right-hand side?

2. How likely would the health state of top chord on the left-hand side be influenced by the degraded state of the top chord on the right-hand side?

3. How likely would the health state of the bottom chord on the left-hand side be influenced by the degraded state of the top chord on the right-hand side?

d) The linguistic scale, given in Table 1, Is provided to the experts.

e) The triangular fuzzy membership function, given in Figure 2, is used to estimate the linguistic analysis numerically. The answers to the three questions are given in Table 2. For example, to Question 1, the most experienced respondent, Expert 2, said unlikely, whilst Expert 1 and 3 said likely and even chance, respectively. Table 2 also shows the triangular fuzzy membership value for each analysis of the experts (in square brackets). Finally, the individual answers are merged by taking account of the level of experience of the respondents, by using Eq. (1). Note that $\beta$ is equal to 0.91 , and $\beta$ has been optimized by using the sensitivity analysis in order to avoid a single member judgment. Therefore, the aggregate result in Table 2 is based on the information retrieved by the whole group of experts, i.e. the analysis of the most experienced respondent is not dominant.

\begin{tabular}{|c|c|c|c|}
\hline Expert & Question 1 & Question 2 & Question 3 \\
\hline Expert 1 & Likely [2, 5/2, 3] & Likely [2, 5/2, 3] & Likely [2, 5/2, 3] \\
\hline Expert 2 & Unlikely [1, 3/2, 2] & Unlikely [1, 3/2, 2] & Very unlikely [1/2, 1, 3/2] \\
\hline Expert 3 & Even chance [3/2, 2, 5/2] & Even chance [3/2, 2, 5/2] & Even chance [3/2, 2, 5/2] \\
\hline Aggregate & Even chance [3/2, 2, 5/2] & Even chance [3/2, 2, 5/2] & Unlikely [1, 3/2, 2] \\
\hline
\end{tabular}

Table 2. Individual and aggregate results.

f) The aggregate answers can be expressed in a fuzzy pairwise comparison matrix. Table 3 shows this matrix, with respect to the influences among the major elements of the bridge. The FAHP is then used to evaluate the weight of the major element on the health state of other 
major elements of the bridge (Eq. (2)), and thus the influence of the parent node (TCR) on the health state of the other major elmenets of the bridge (TCR, TCL, BCR and BCL) is:

$$
w=(0.2759,0.2759,0.2241,0.2241)^{T}
$$

\begin{tabular}{|l|l|l|l|l|}
\cline { 2 - 5 } \multicolumn{1}{c|}{} & $T C R$ & $B C R$ & $T C L$ & $B C L$ \\
\hline$T C R$ & {$[1,1,1]$} & {$[1,1,1]$} & {$[1,1,1]$} & {$[1,3 / 2,2]$} \\
\hline$B C R$ & {$[1,1,1]$} & {$[1,1,1]$} & {$[1,3 / 2,2]$} & {$[1,1,1]$} \\
\hline$T C L$ & {$[1,1,1]$} & {$[1 / 2,2 / 3,1]$} & {$[1,1,1]$} & {$[1,1,1]$} \\
\hline$B C L$ & {$[1 / 2,2 / 3,1]$} & {$[1,1,1]$} & {$[1,1,1]$} & {$[1,1,1]$} \\
\hline
\end{tabular}

Table 3. A fuzzy pairwise comparison matrix for major elements.

g) The consistency of the expert analysis is verified by evaluating the $C R$ :

$$
C R=\frac{C l}{R l}=\frac{4.0414-4}{4} \cdot \frac{1}{0.9000}=0.0154
$$

where $\lambda_{\max }=4.0414$ is retrieved by using the maximum centroid of area method to defuzzify the pairwise matrix of Table $3 . R I$ is equal to 0.9 , and is obtained by using literature [Wang and Elhag, 2006]. Since $C R$ is lower than 0.1 , the analysis is consistent.

h) In this final stage of the CPT development, the first step is to define the probability of having a child node in a state $i$, conditional on the knowledge that its parent nodes are in the healthy state $(\mathrm{H}), P\left(x_{i} \mid \bigcap_{k=1}^{N} Y^{k}=y_{k}=1\right)$, with $i=\mathrm{H}, \mathrm{PD}, \mathrm{SD}$. In this paper, we assume that the health state of each element, when there is no degradation of an element in the parent node, is equal to:

$P\left(x_{i} \mid \bigcap_{k=1}^{N} Y^{k}=y_{k}=1\right)=\left[P\left(x_{H} \mid \bigcap_{k=1}^{N} Y^{k}=y_{k}=1\right)=0.95, P\left(x_{P D} \mid \bigcap_{k=1}^{N} Y^{k}=y_{k}=1\right)=0.025\right.$, $P\left(x_{S D} \mid \bigcap_{k=1}^{N} Y^{k}=y_{k}=1\right)=0.025$ ]. For example, the CPT of the TCR_1 node of the BBN is shown in Table 4, which has two parent nodes, TCR and TCL. There are 27 entries in the CPT as each of the three elements has three states $\left(3^{3}\right)$. The first column gives the probabilities of $T C R \_1$ states, conditional on the knowledge that both parents are in the healthy state. 
Furthermore, the rest of entries are defined using Eq. (5), where $p_{i}$ and $f_{p_{m}}$ depend on the health state of the parent nodes $k$ :

$$
\begin{aligned}
& p_{i}= \begin{cases}{[1,0.7,0.3]^{T}} & \text { if } k=P D \\
{[1,0.25,0.75]^{T}} & \text { if } k=S D\end{cases} \\
& f_{p_{m}}= \begin{cases}1 & \text { if } k=H \text { or } P D \\
1.1 & \text { if } k=S D\end{cases}
\end{aligned}
$$

For example, Eq. (9) shows that $p_{i}$ is equal to 0.7 if $T C R \_1$ and the parent node are in the PD state. In this way, the decrease of $P\left(T C R_{-} 1_{i} \mid \bigcap_{k=1}^{N} Y^{k}=y_{k}=1\right)$ is shared between the states PD and SD, and the whole probability is normalized to 1 ; an example of this process is presented in Eq. (11) and Eq. (12).

Note that the weight of the parent nodes on the child node are equal to $w=(0.2759,0.2241)$, as shown in step $f)$. For example, the two columns with text in bold in Table 4 are computed by using Eq. (5), resulting in Eq. (11) (where the number of degraded elements is equal to $1, M=1$ ), and Eq. (12), (where the number of degraded elements is equal to 2, $M=2)$ :

$$
\left\{\begin{array}{l}
P\left(T C R_{-} 1_{H} \mid Y_{k=P}^{T C R}, Y_{k=H}^{T C L}\right)=0.95+0.95 \cdot 0.2759 \cdot 1 \cdot(-1) \cdot 1=0.6879 \approx 0.69 \\
P\left(T C R_{-} 1_{P D} \mid Y_{k=P D}^{T C R}, Y_{k=H}^{T C L}\right)=0.025+0.95 \cdot 0.2759 \cdot 0.7 \cdot(1) \cdot 1=0.2085 \approx 0.21 \\
P\left(T C R_{-} 1_{S D} \mid Y_{k=P D}^{T C R}, Y_{k=H}^{T C L}\right)=0.025+0.95 \cdot 0.2759 \cdot 0.3 \cdot(1) \cdot 1=0.1036 \approx 0.10
\end{array}\right.
$$

$$
\left\{\begin{array}{l}
P\left(T C R_{-} 1_{H} \mid Y_{k=S D}^{T C R}, Y_{k=P D}^{T C L}\right)=0.95+0.95 \cdot(0.2759 \cdot 1.1+0.2241 \cdot 1) \cdot 1 \cdot(-1)=0.4480 \approx 0.45 \\
P\left(T C R_{-} 1_{P D} \mid Y_{K=S D}^{T C R}, Y_{K=P D}^{T C L}\right)=0.025+0.95 \cdot(0.2759 \cdot 1.1+0.2241 \cdot 1) \cdot 0.25 \cdot(1)=0.1503 \approx 0.15 \\
P\left(T C R_{-} 1_{S D} \mid Y_{K=S D}^{T C R}, Y_{K=P D}^{T C L}\right)=0.025+0.95 \cdot(0.2759 \cdot 1.1+0.2241 \cdot 1) \cdot 0.75 \cdot(1)=0.4009 \approx 0.40
\end{array}\right.
$$

\begin{tabular}{|c|c|c|c|c|c|c|c|c|c|c|}
\cline { 2 - 11 } \multicolumn{1}{c|}{} & TCL & \multicolumn{3}{c|}{$\mathrm{H}$} & \multicolumn{3}{c|}{ PD } & \multicolumn{3}{c|}{ SD } \\
\cline { 2 - 11 } & TCR & $\mathrm{H}$ & $\mathrm{PD}$ & $\mathrm{SD}$ & $\mathrm{H}$ & $\mathrm{PD}$ & $\mathrm{SD}$ & $\mathrm{H}$ & $\mathrm{PD}$ & $\mathrm{SD}$ \\
\hline \multirow{3}{*}{$T C R \_1$} & $\mathrm{H}$ & 0.95 & $\mathbf{0 . 6 9}$ & 0.63 & 0.73 & 0.47 & $\mathbf{0 . 4 5}$ & 0.69 & 0.43 & 0.38 \\
\cline { 2 - 11 } & $\mathrm{PD}$ & 0.025 & $\mathbf{0 . 2 1}$ & 0.11 & 0.16 & 0.36 & $\mathbf{0 . 1 5}$ & 0.08 & 0.16 & 0.17 \\
\cline { 2 - 11 } & $\mathrm{SD}$ & 0.025 & $\mathbf{0 . 1 0}$ & 0.26 & 0.01 & 0.17 & $\mathbf{0 . 4 0}$ & 0.21 & 0.41 & 0.45 \\
\hline
\end{tabular}

Table 4. The resulting CPT for the TCR_1 node 


\subsubsection{Results of the BBN method application to detect steel truss bridge degradation}

The performance of the proposed BBN method is evaluated by analysing the 28 degradation scenarios, presented in Section 3.1.1. In the method, the displacements of the bridge during the 28 degraded scenarios are used as an input to the BBN. The displacement of the bridge elements is used as an input to the sensor nodes of the BBN. In this way, the health state of the minor elements of the bridge is assessed based on the value of the displacement of the bridge elements. In fact, the displacement of the bridge is directly related to the health state of the bridge, i.e. a degradation of the bridge health state is expected to be reflected by a change of the bridge element displacement, which generally increases if a given load on the bridge is defined. When the health state of the minor elements is selected, the health state of all bridge elements is updated accordingly. The scenarios are chosen in a random order. The method is used to identify the deteriorated elements and the level of deterioration, i.e. the elements and their states are ranked in terms of their occurrence probabilities.

\begin{tabular}{|c|c|c|c|c|}
\hline $\begin{array}{c}\text { Degradation } \\
\text { scenarios }\end{array}$ & $\begin{array}{c}\text { Number } \\
\text { of } \\
\text { scenarios }\end{array}$ & $\begin{array}{c}\text { Number of correct } \\
\text { identifications of } \\
\text { the degraded major } \\
\text { elements }\end{array}$ & $\begin{array}{c}\text { Number of correct } \\
\text { identifications of } \\
\text { the degraded minor } \\
\text { elements }\end{array}$ & $\begin{array}{c}\text { Number of false } \\
\text { identifications of the } \\
\text { degraded minor } \\
\text { elements }\end{array}$ \\
\hline $\begin{array}{c}\text { A Single } \\
\text { element }\end{array}$ & 22 & $18 / 22$ & $17 / 22$ & $10 / 22$ \\
\hline $\begin{array}{c}\text { Multiple } \\
\text { elements }\end{array}$ & 6 & $10 / 12$ & $15 / 18$ & $7 / 18$ \\
\hline Total & 28 & $28 / 34$ & $32 / 40$ & $17 / 44$ \\
\hline
\end{tabular}

Table 5. Deterioration detection on the steel truss bridge using the BBN method

The results are presented in Table 5, and can be discussed as follows:

- Major Elements. Good accuracy in detecting the degraded major element(s) of the bridge is demonstrated. When a single element is degraded, the BBN is able to correctly identify 18 out of 22 major elements, which contain a degraded minor element (accuracy of $82 \%$ ). When multiple minor elements are degraded, the BBN correctly identifies 10 out of 12 major elements that contain degraded minor element(s). Note that 12 is the number of major elements that are degraded during the six scenarios with multiple elements.

Minor Elements. Good accuracy is also shown in detecting the degraded minor elements, i.e. 32 out of 40 minor elements are correctly detected. When multiple minor elements are degraded, the BBN correctly identifies 15 out of 18 minor degrading elements. Note that 18 is the number of minor elements that are degraded during the six scenarios with multiple elements. 
However, there are some misclassifications obtained:

- Major elements. 5 scenarios (out of 28) are misclassified. These scenarios are situations when the element at the end of the bottom chord, i.e. at the joint, is degraded. These joints represent the support of the bridge, as shown in Figure 3, and thus the displacement of all bridge elements is slightly influenced by the degradation of these joints. Therefore, using the BBN method it is impossible to identify this degradation as it only results in small changes in behaviour. This is a commonly observed issue for some SHM analyses, when the degradation of the lateral parts of the bridge is hard to identify [Vagnoli et al., 2018]. There was also a misclassification case between the bottom and the top chords; this could be because, while developing the BBN model, high influence among minor (major) elements of the bridge was assumed.

- Minor Elements. 3 minor elements were misclassified when multiple minor elements of both bottom chords have degraded; in this case the mid-span of the bridge as the element that has degraded has been identified by the method. In addition, 17 elements were erroneously identified as degraded; again, this could be because of the assumed influence between neighbouring elements.

\subsubsection{The post-tensioned concrete bridge and degradation scenarios}

In this case study, the focus is placed on illustrating how the BBN method can be applied for detecting degradation of bridge elements and for continuous monitoring of their health states, using in-field data of bridge acceleration.

This in-field post-tensioned concrete bridge [Siringoringo et al., 2013] has a main span of $32 \mathrm{~m}$ and two side spans of $12 \mathrm{~m}$, and its width is $6.6 \mathrm{~m}$ (Figure 7a). The bridge was subject to a progressive damage test before being demolished, i.e. the infrastructure of the bridge was intentionally damaged in order to study how the bridge behaves in different health states. The main excitation source of the bridge was changing environmental conditions.

The acceleration of the bridge was monitored by a measurement system made of two reference sensors, which were kept fixed throughout the duration of the progressive damage test. The sampling rate of the sensors was $100 \mathrm{~Hz}$, and they were installed at locations, shown by circles in Figure 7b. A progressive damage test was performed by cutting a pier of the bridge, as shown in Figure 7c. The 
bridge acceleration of during five different situations, related to the state of the bridge, was monitored (Figure 7c):

- Situation 0: the healthy state, i.e. the progressive damage test has not started yet.

- Situation 1: the left pier was cut by $5 \mathrm{~cm}$ and a steel column was installed to provide a temporary support of the bridge.

- Situation 2: the steel column was lowered by $1 \mathrm{~cm}$ and the bridge deck settled at $1 \mathrm{~cm}$ lower than its starting position.

- Situation 3: the steel column was further lowered by $1 \mathrm{~cm}$ and the bridge deck settled at $2 \mathrm{~cm}$ lower than its starting position.

- Situation 4: the steel column was lowered by $3 \mathrm{~cm}$ and the bridge deck settled at $2.7 \mathrm{~cm}$ lower than its starting position.

It is worth mentioning that the health states of the bridge can be represented as follows: $i$ ) healthy state of the bridge, when the bridge is in situation 0 (healthy state in Figure 7c); ii) partially degraded state, when the bridge is in situation 1 and situation 2 (state 1 and state 2 in Figure 7c), due to the fact that the steel column holds the bridge or is slightly lowered; iii) severely degraded state, when the bridge is in situation 3 and situation 4 (state 3 and state 4 in Figure 7c), due to the fact that the steel column is significantly lowered, and the bridge deck position is modified accordingly. 


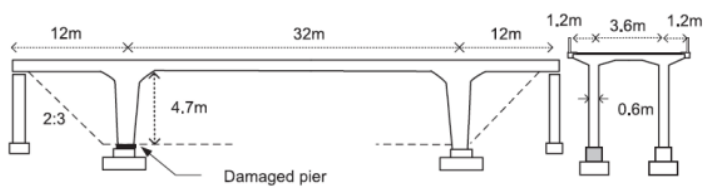

(a)

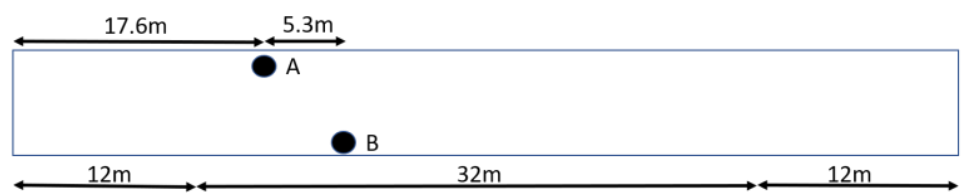

(b)
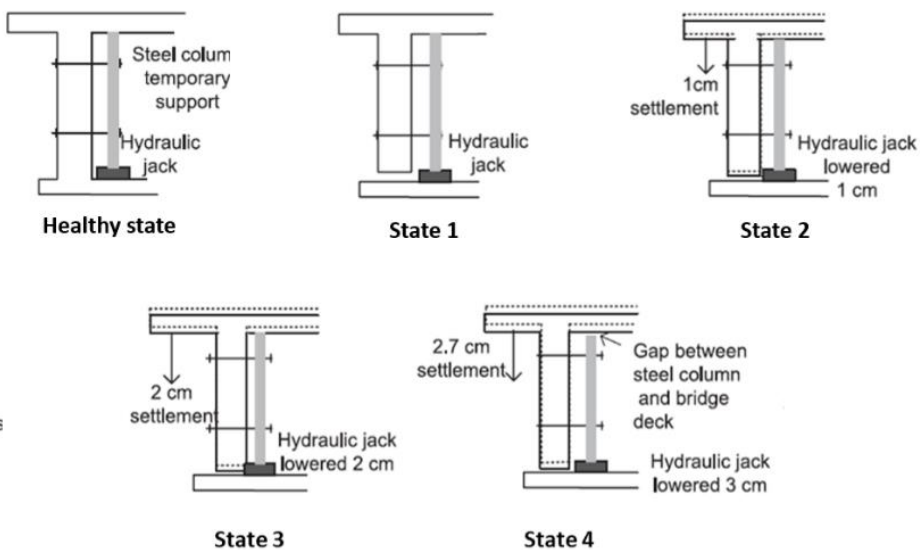

(c)

Figure 7. The post-tensioned concrete bridge

\subsubsection{Building the BBN for the concrete bridge}

1. In this case study, the major elements are the three spans of the bridge (i.e. the main span and left and right side spans) and the piers. The deck, which is made of these three spans, is of total of $56 \mathrm{~m}$ long and is divided into 10 minor elements, of $5.6 \mathrm{~m}$. The condition of the deck represents the condition of the whole bridge.

2. Since the deck is made of three spans, three nodes in the BBN model represent these major elements, and 10 nodes represent the minor elements, as shown in Figure 8. The minor elements are denoted as $E \_j$, where $j=1,2, \ldots, 10$. In addition, the nodes $E \_2$ and $E \_3$ represent the minor elements at the left pier, and nodes $E_{-} 8$ and $E_{-} 9$ represent the minor elements at the right pier. The three major elements influence the health state of the whole deck, and, consequently, they are parents of the Deck node. 


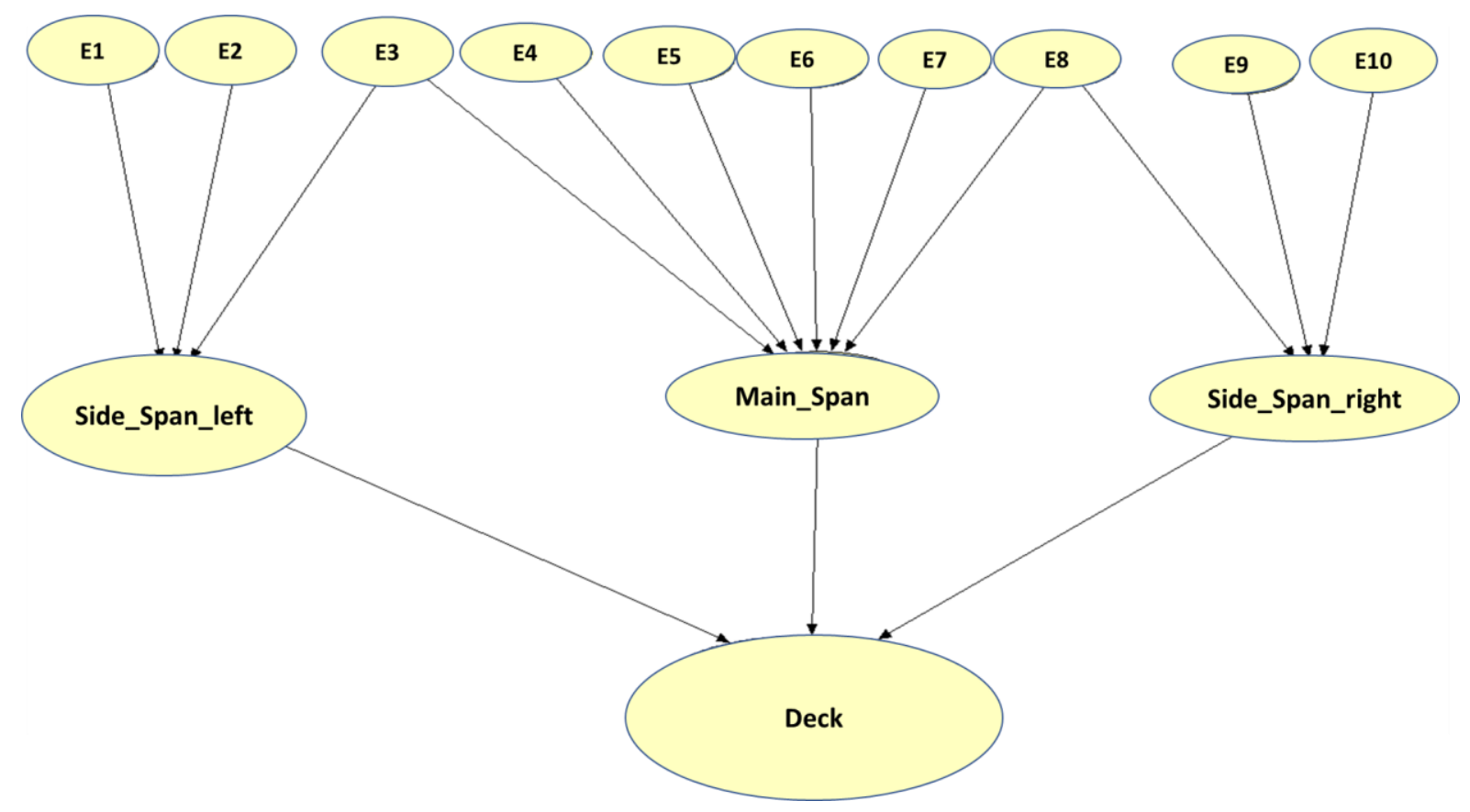

Figure 8. The BBN model for the post-tensioned concrete bridge

In addition, interdependencies among neighbouring major/minor elements are also included, and further nodes, ending with “_1", are introduced, as shown in Figure 9.

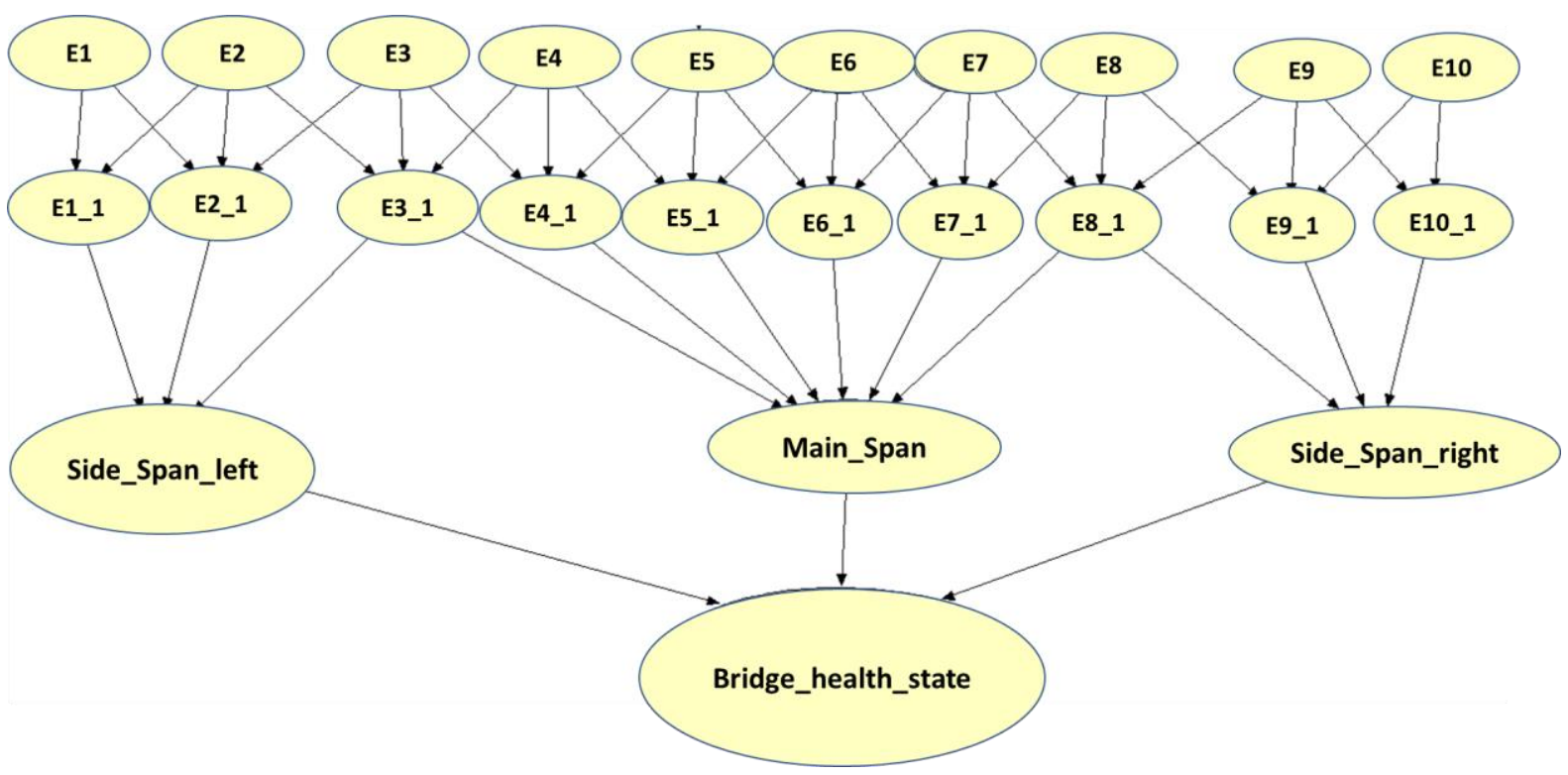

Figure 9. The BBN model with additional interdependencies.

3. The five states defined during the progressive damage test (described in 3.2.1) are grouped into three health states: $i$ ) a healthy state, situation 0 from the in-field test; ii) a partially degraded state, situations 1 and 2; iii) a severely degraded state, situations 3 and 4 . 
4. The measurement system of the bridge is represented by two accelerometers that are installed at $17.6 \mathrm{~m}$ and $22.6 \mathrm{~m}$ from the left end of the bridge, as shown in Figure 7. The two sensor nodes are added to the BBN model at respective minor elements, as depicted in Figure 10.

Note that the CPTs are developed following the same approach as in the first case study, presented in Section 3.1.4.

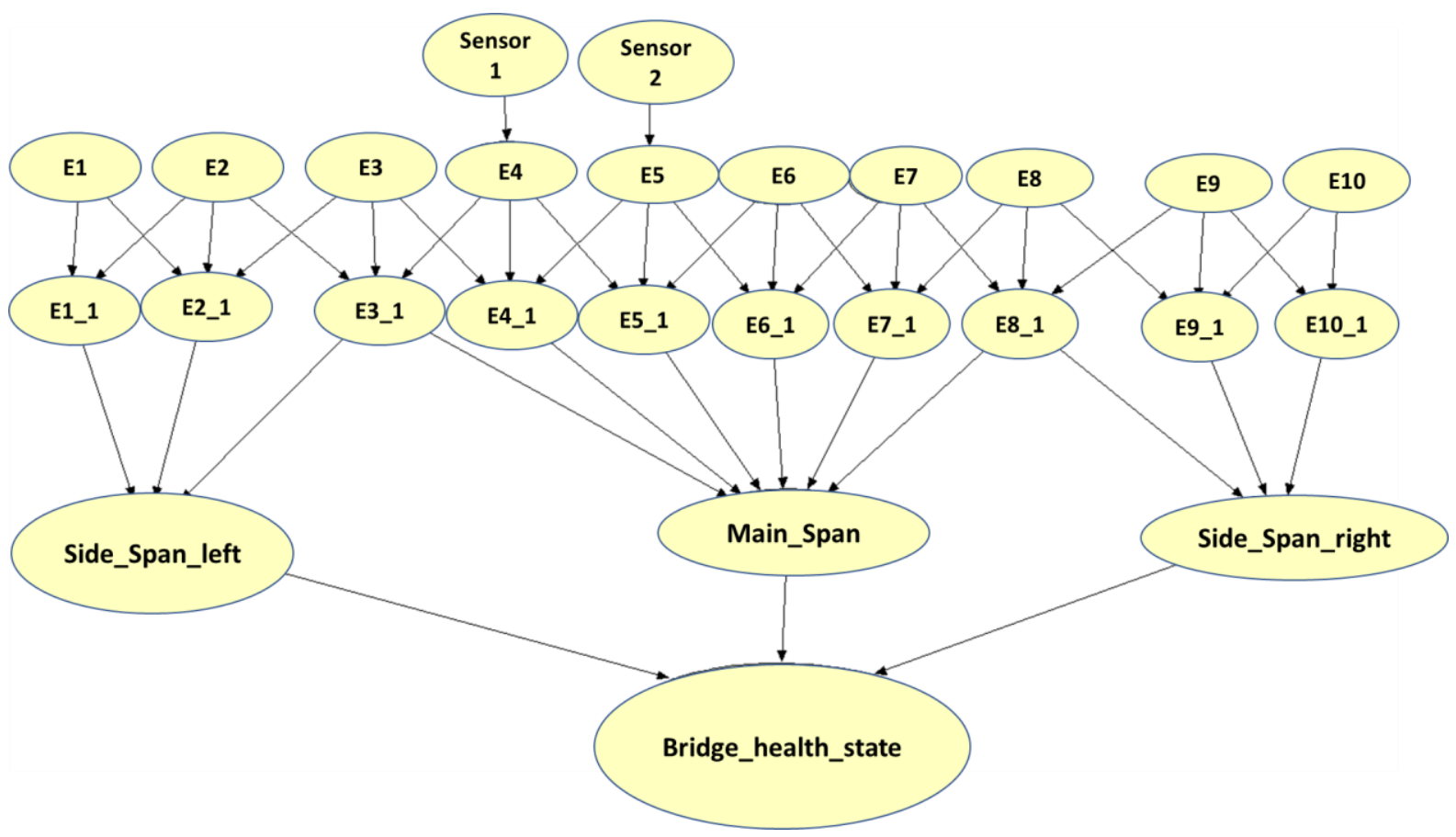

Figure 10. The final BBN model

\subsubsection{Results of the BBN method application to detect concrete bridge degradation and to monitor its health state}

The performance of the method is evaluated by analysing the five degradation scenarios, presented in Section 3.2.1. In the method, bridge acceleration data is used as an input to the BBN. Particularly, the acceleration data are firstly subjected to a pre-processing analysis with the aim of removing the noise from the acceleration data and lumping the data into a health indicator [Moughty and Casas, 2017]. Then, the post-processed acceleration data are used as an input to the sensor nodes. The health state of the minor bridge elements is then assessed by evaluating the magnitude of the health indicator of the bridge, which is related to the health state of the bridge subject to an external excitation: the higher the bridge acceleration, the higher the degradation of the bridge elements. The results show how the health state of the bridge and its elements changes over time, i.e. probability of being in the healthy state deceases and probabilities of being in the partially and severely degraded states increase when a progressive damage test is carried out. 


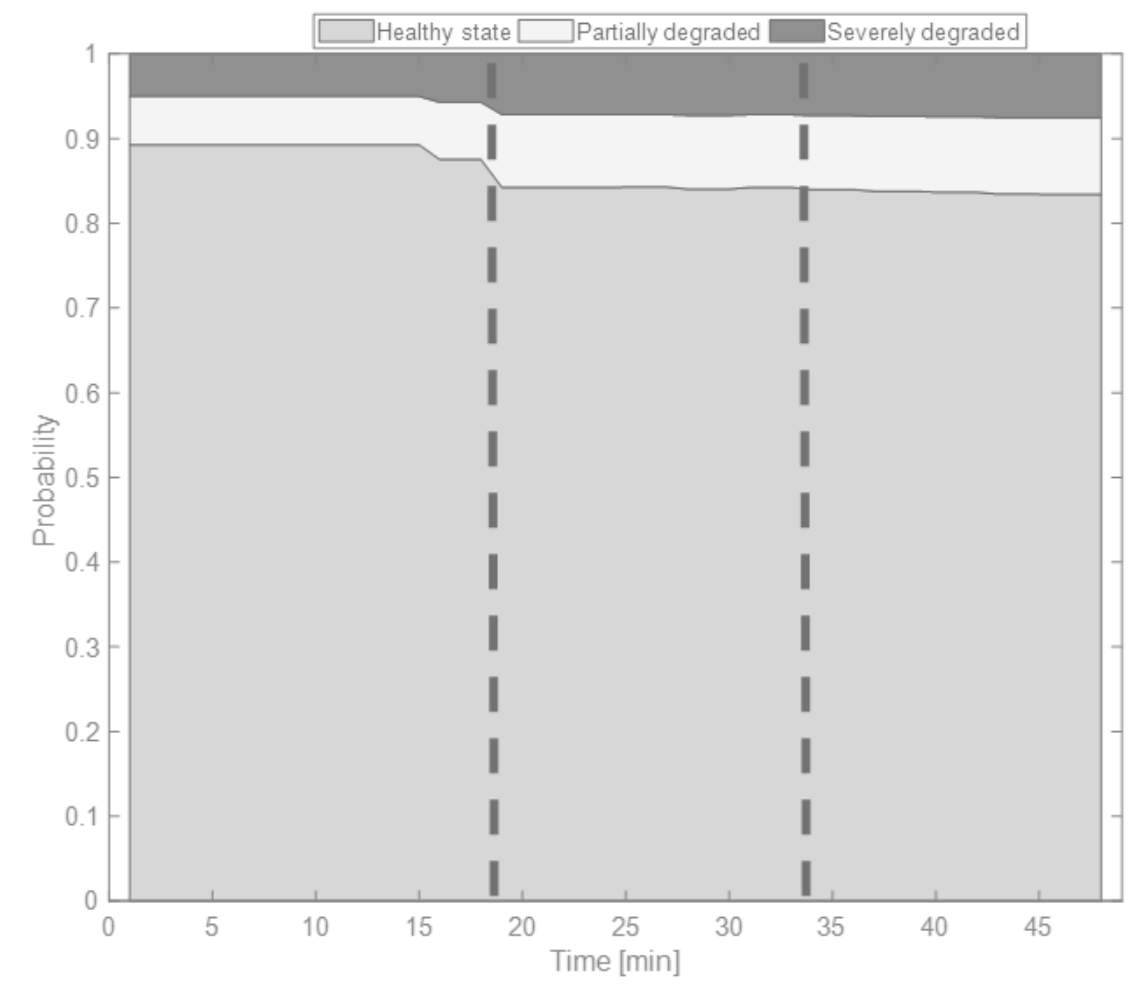

Figure 11. Evolution of the health state of the deck over the duration of the damage test

For example, Figure 11 shows the evolution of the health state of the deck over time. At the beginning, the bridge is in the healthy state before any damage occurs, represented as the grey area in Figure 11. There is an increase in the probability of the partially degraded state and the severely degraded state at around time $15 \mathrm{~min}$, represented by light grey and dark grey areas, respectively. This is due to noise in the data. The dashed vertical lines represent the time when the bridge is damaged by cutting the left pier of the bridge. The first vertical dashed line at a time of $19 \mathrm{~min}$ represents the occurrence of the partially degraded state (i.e. damage described by States 1 and 2), whereas the second dashed line at a time of $34 \mathrm{~min}$ represents the occurrence of the severely degraded state (i.e. damage described by States 3 and 4). At a time of $19 \mathrm{~min}$, the probability of being in the partially and severely degraded states increases significantly, and it is higher than at the beginning of the damage test. A further slight increase in these probabilities is observed at time of $34 \mathrm{~min}$ when further damage is inflicted. This small example illustrates that the BBN method can be used to monitor the health state over time when unexpected bridge behaviour is detected as soon as it occurs. Figure 11 also shows that the largest area of the probability belongs to the healthy state of the bridge: this result can be due to both the definition of the CPTs and the structure of the BBN. In terms of the definition of the CPTs, such a result is possible if a low probability is assumed for the influence of minor components to the overall bridge health state, i.e. the degrading state of the bridge minor elements has a low impact on the health state of the whole 
bridge. In terms of the structure of the BBN, the evidence about the degrading minor elements is combined with the evidence about healthy elements and due to the structure of the BBN the healthy state has more influence on the outcome. This outcome can be improved by using a more robust definition of the CPTs, e.g. merging the expert knowledge with the analysis of the bridge behaviour, and reducing the number of nodes in the BBN.

As shown in the case study in Section 3.1, the BBN method can also be used to detect deterioration of the bridge and its elements, i.e. find the most likely location of the deterioration.

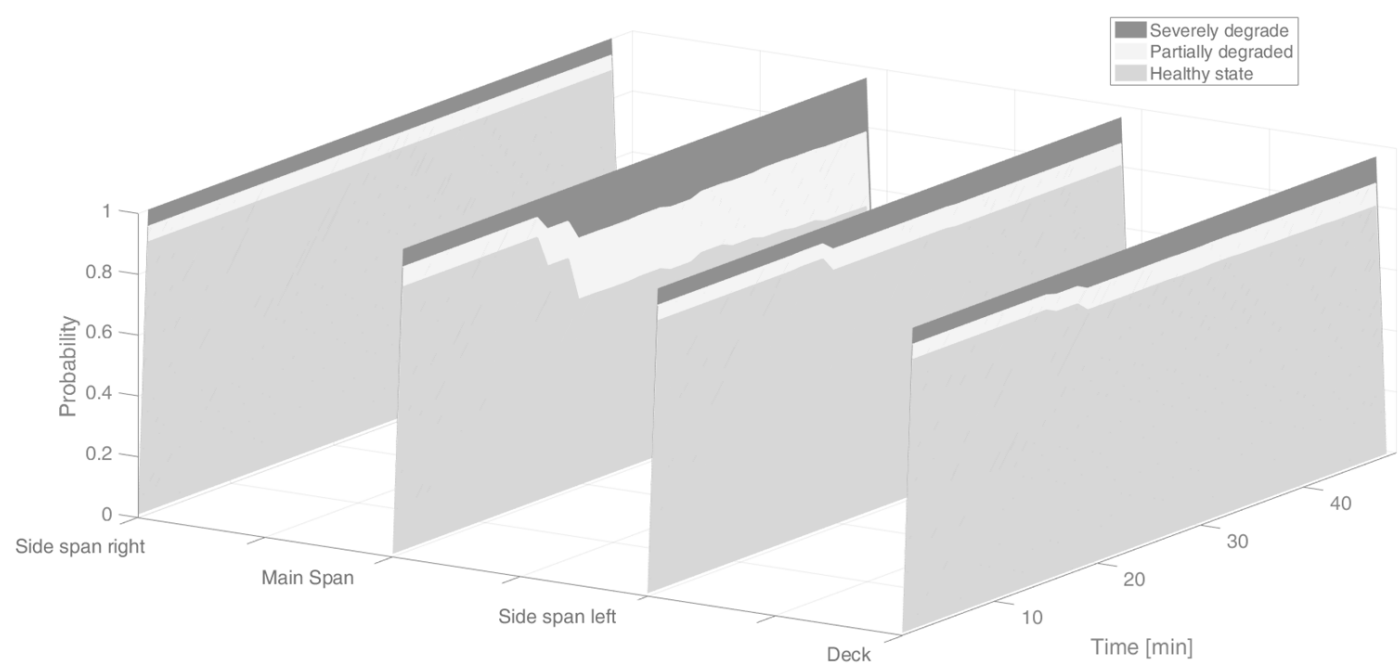

Figure 12. Evolution of the health state of the deck and its parent nodes over the duration of the damage test

For example, in addition to the evolution of the health state of the deck, Figure 12 shows the evolution of the health states for the parent nodes of the Deck node: the main span, the side span on the left and the side span on the right. It identifies that at a time of $19 \mathrm{~min}$ the location of the degradation is the main span. It is more degraded than the other two major elements, because the light grey and dark grey areas of the main span are larger than those areas of the side span on the left and on the right. This illustrates that the BBN method can identify the location correctly. Note that the damaged left pier is represented by two nodes in the BBN, which influence the main span directly and have some influence to the side span on the left. Therefore, the light grey and dark grey of the side span on the left are larger than for the side span on the right, but not as large as for the main span. 

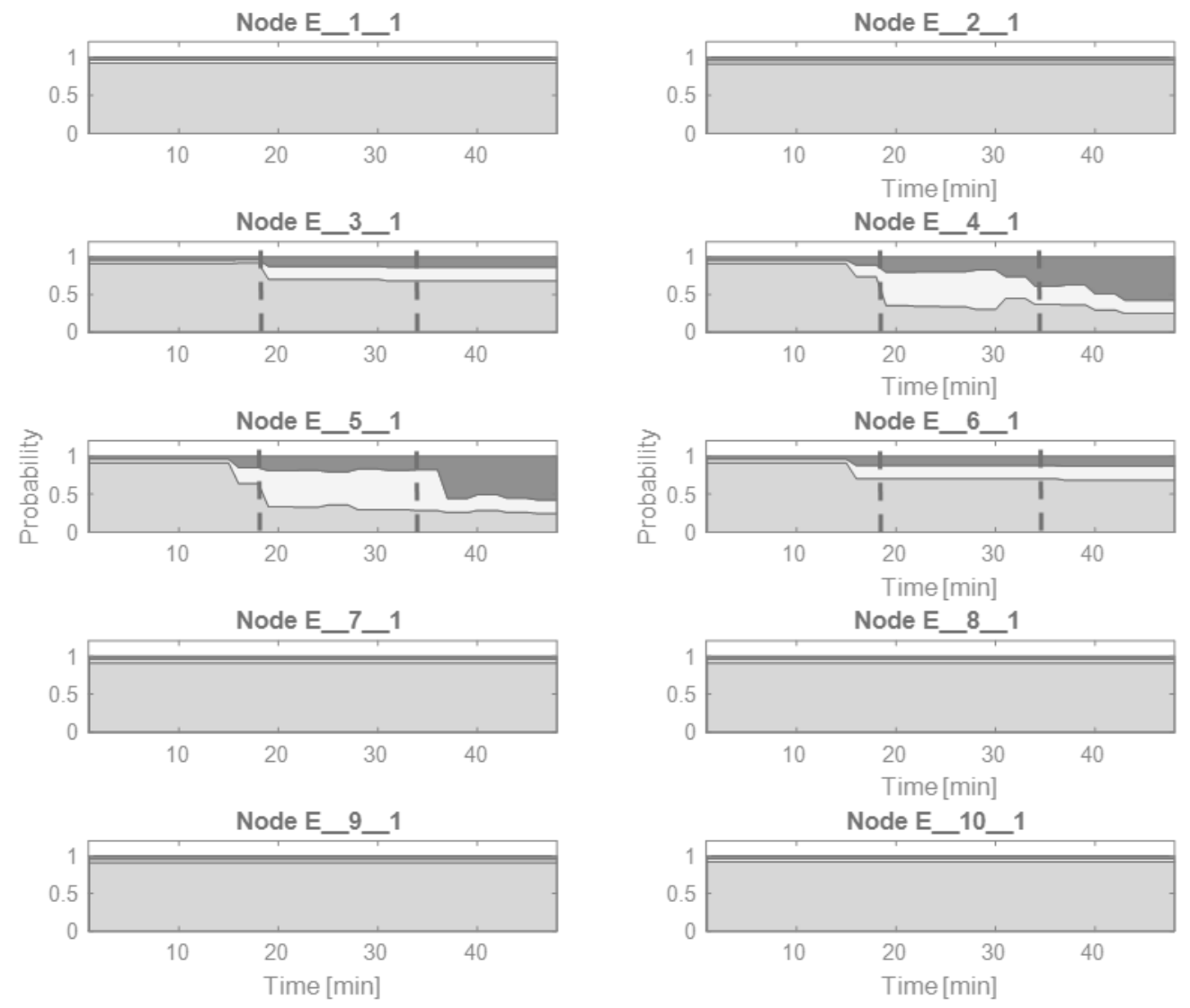

Figure 13. Evolution of the health state of the minor elements over the duration of the damage test

At the minor element level, it can be seen in Figure 13 that the elements $E \_4 \_1$ and $E \_5 \_1$ have been identified as the most likely location of the damage. These two nodes also represent the locations of the sensors. Since the left pier was damaged, its associated nodes are the elements $E \_3 \_1$ and $E \_4 \_1$. Therefore, this example illustrates that the location has been identified only partially, i.e. there are some changes in the light grey and dark grey areas for $E \_3 \_1$ but they are not as large as for $E \_4 \_1$ and E_5_1. At the same time, Figure 13 shows that different health states of the bridge are identified by the changing states of elements $E_{-} 4 \_1$ and $E \_5 \_1$ : when the bridge experiences situation 1 and 2 (partially degraded states at min 19) the health state of the bridge elements worsens; similarly, when the bridge experiences situation 3 and 4 , i.e. the bridge enters into the severely degraded states, the health state of elements $E \_4 \_1$ and $E \_5 \_1$ worsens significantly. For the element $E \_6 \_1$, some changes in the light grey and dark grey are also seen; this could be because of the assumed influence between the neighbouring elements.

In terms of the accuracy of the method, demonstrated in the two case studies, it should be said, that better results could potentially be achieved by increasing the number of nodes, used to represent bridge 
elements. This could improve the ability to locate the degradation on the bridge, but it would come with an expense of having to develop and analyse larger and more detailed BBN models. Furthermore, the accuracy of the CPTs could be enhanced by using data, collected by a bridge sensor system, in addition to, or instead of, expert knowledge.

\section{Conclusion}

In this paper, a novel BBN method has been proposed for bridge degradation detection and for evaluation of the bridge health state. The method has been applied in two case studies. In the first case study, a steel truss bridge was modelled using an FEM model, and effects of degradation scenarios of individual and multiple element deterioration have been obtained from the FEM model. Using the proposed method the degraded elements of the bridge have been identified correctly in most cases. In the second case study, instead of using the FEM model, acceleration data of an in-field post-tensioned concrete bridge has been used as an input to the BBN model. Again, bridge elements that were most likely to have deteriorated were identified. The method was also used to find the point in time when the change in the behaviour of the bridge has occurred. Overall, using the proposed BBN method, evolution of the health state of the bridge and its elements over the duration of the damage test has been obtained in a novel way.

The BBN method has shown some good performance in both case studies. However, some misclassifications of deteriorated elements have occurred, potentially due to a number of factors, such as noisy in-field data, the level of detail in the BBN model and the method adopted for the CPT development. Better results could potentially be achieved by increasing the number of nodes, used to represent bridge elements in the BBN model. This could improve the ability to locate the degradation on the bridge but it would come with the expense of having to develop and analyse larger and more detailed BBN models. Furthermore, the accuracy of the CPTs could be enhanced by using data, collected by a bridge sensor system, instead of expert knowledge. Future work could include an exploration of the method application to different types of bridges and different deterioration mechanisms. This work should be further developed using other case studies of instrumented bridges. 


\section{Funding}

This project has received funding from the European Union's Horizon 2020 research and innovation programme under the Marie Skłodowska-Curie grant agreement No. 642453.

\section{Disclosure statement}

No potential conflict of interest was reported by the authors.

\section{References}

Ahmadi, H.R., Anvari, D., "New damage index based on least squares distance for damage diagnosis in steel girder of bridge's deck", Structural Control and Health Monitoring, 2018, 25 (10), art. no. e2232

Alves, V., Cury, A., Roitman, N., Magluta, C., Cremona, C., "Structural modification assessment using supervised learning methods applied to vibration data", Engineering Structures, 2015, 99, pp. 439-448.

Arangio, S., Beck, J.L., "Bayesian neural networks for bridge integrity assessment", Structural Control and Health Monitoring, 2012, 19 (1), pp. 3-21.

Attema, T., Kosgodagan Acharige, A., Morales-Nápoles, O., Maljaars, J., "Maintenance decision model for steel bridges: a case in the Netherlands", Structure and Infrastructure Engineering, 2017, 13 (2), pp. 242-253.

Attoh-Okine, N.O., Bowers, S., "A Bayesian belief network model of bridge deterioration", Proceedings of the Institution of Civil Engineers: Bridge Engineering, 2006, 159 (2), pp. 69-76.

Casas, J.R., Moughty, J. J., "Bridge Damage Detection Based on Vibration Data: Past and New Developments", Front. Built Environ. 3:4., 2017, doi: 10.3389/fbuil.2017.00004.

Bao, Y., Valipour, M., Meng, W., Khayat, K.H. and Chen, G., Distributed fiber optic sensor-enhanced detection and prediction of shrinkage-induced delamination of ultra-high-performance concrete overlay. Smart Materials and Structures, 2017, 26(8), p.085009

Dağdeviren, M., Yüksel, I., "Developing a fuzzy analytic hierarchy process (AHP) model for behaviorbased safety management", Information Sciences, 2008, 178 (6), pp. 1717-1733.

Dowling, J., Obrien, E.J., González, A., "Adaptation of Cross Entropy optimisation to a dynamic Bridge WIM calibration problem", Engineering Structures, 2012, 44, pp. 13-22.

Elmasry, M., Hawari, A., Zayed, T., "Defect based deterioration model for sewer pipelines using bayesian belief networks", Canadian Journal of Civil Engineering, 2017, 44 (9), pp. 675-690.

European Commission, "EU transport in figures (statistical pocketbook)", Brussels: European Commission, 2012

Fan, W., Qiao, P., "Vibration-based damage identification methods: A review and comparative study", Structural Health Monitoring, 2011, 10 (1), pp. 83-111.

Franchin, P., Lupoi, A., Noto, F., Tesfamariam, S., "Seismic fragility of reinforced concrete girder bridges using Bayesian belief network", Earthquake Engineering and Structural Dynamics, 2016, 45 (1), pp. 29-44.

Frangopol, D.M., Saydam, D., Kim, S., "Maintenance, management, life-cycle design and performance of structures and infrastructures: a brief review", Structure and Infrastructure Engineering, 2012, 8 (1), pp. 1-25.

Gentile, C., Saisi, A., "Continuous dynamic monitoring of a centenary iron bridge for structural modification assessment", Frontiers of Structural and Civil Engineering, 2015, 9 (1), pp. 26-41.

Holický, M., Marková, J., Sýkora, M., "Forensic assessment of a bridge downfall using Bayesian networks", Engineering Failure Analysis, 2013, 30, pp. 1-9.

Hester, D., J. Brownjohn, M. Bocian and Y. Xu. "Low Cost Bridge Load Test: Calculating Bridge Displacement from Acceleration for Load Assessment Calculations." Engineering Structures 143 (2017): 358-374.

Hsu, T.-Y., Loh, C.-H., "Damage detection accommodating nonlinear environmental effects by nonlinear principal component analysis", Structural Control and Health Monitoring, 2010, 17 (3), pp. 338-354.

Jensen, F.V., Nielsen, T. D., (2007). "Bayesian Networks and Decision Graphs", Information Science and Statistics, Springer. 
Kabir, G., Sadiq, R., Tesfamariam, S., "A fuzzy Bayesian belief network for safety assessment of oil and gas pipelines", Structure and Infrastructure Engineering, 2016, 12 (8), pp. 874-889.

Kim, J.-T., Park, J.-H., Lee, B.-J., "Vibration-based damage monitoring in model plate-girder bridges under uncertain temperature conditions", Engineering Structures, 2007, 29 (7), pp. 1354-1365.

Kreislova, K., Geiplova, H., "Evaluation of corrosion protection of steel bridges", Procedia Engineering, 2012, 40, pp. 229-234.

Loughney, S., Wang, J., "Bayesian network modelling of an offshore electrical generation system for applications within an asset integrity case for normally unattended offshore installations", Proceedings of the Institution of Mechanical Engineers, Part M: Journal of Engineering for the Maritime Environment, 2017, p.1475090217704787.

Martínez-Martínez, L.H., Delgado-Hernández, D.J., de-León-Escobedo, D., Flores-Gomora, J., Arteaga-Arcos, J.C., "Woody debris trapping phenomena evaluation in bridge piers: A Bayesian perspective", Reliability Engineering and System Safety, 2017, 161, pp. 38-52.

Mehrjoo, M., Khaji, N., Moharrami, H., Bahreininejad, A., "Damage detection of truss bridge joints using Artificial Neural Networks", Expert Systems with Applications, 2008, 35 (3), pp. 1122-1131.

Morales-Nápoles, O., Delgado-Hernández, D.J., De-León-Escobedo, D. and Arteaga-Arcos, J.C., " $A$ continuous Bayesian network for earth dams' risk assessment: methodology and quantification", Structure and Infrastructure Engineering, 2014, 10(5), pp.589-603.

Moughty, J.J., Casas, J.R., "A state of the art review of modal-based damage detection in bridges: Development, challenges, and solutions", Applied Sciences (Switzerland), 2017, 7 (5), art. no. 5100. Mustafa, S., Matsumoto, Y., "Bayesian Model Updating and Its Limitations for Detecting Local Damage of an Existing Truss Bridge", Journal of Bridge Engineering, 22 (7), art. no. 04017019.

Ni, Y.-C., Zhang, Q.-W., Liu, J.-F., (2019). "Dynamic Property Evaluation of a Long-Span CableStayed Bridge (Sutong Bridge) by a Bayesian Method", International Journal of Structural Stability and Dynamics, 2017, 19 (1), art. no. 1940010.

Ni, Y.Q., Xia, H.W., Wong, K.Y., Ko, J.M., "In-service condition assessment of bridge deck using long-term monitoring data of strain response" Journal of Bridge Engineering, 2012, 17 (6), pp. 876885.

Psimoulis, P.A., Stiros, S.C., "Measuring deflections of a short-span railway bridge using a robotic total station", Journal of Bridge Engineering, 2013, 18 (2), pp. 182-185.

Rafiq, M.I., Chryssanthopoulos, M.K., Sathananthan, S., "Bridge condition modelling and prediction using dynamic Bayesian belief networks", Structure and Infrastructure Engineering, 2015, 11 (1), pp. 38-50.

Rao, A.S., Lepech, M.D., Kiremidjian, A., "Development of time-dependent fragility functions for deteriorating reinforced concrete bridge piers", Structure and Infrastructure Engineering, 2017, 13 (1), pp. 67-83.

Rice, J.A., Mechitov, K.A., Sim, S.H., Spencer Jr., B.F., Agha, G.A., "Enabling framework for structural health monitoring using smart sensors", Structural Control and Health Monitoring, 2011, 18 (5), pp. 574-587, 2011.

Sanayei, M., Khaloo, A., Gul, M., Necati Catbas, F., "Automated finite element model updating of a scale bridge model using measured static and modal test data", Engineering Structures, 2015, 102, pp. 66-79.

Siringoringo, D.M., Fujino, Y., Nagayama, T., "Dynamic characteristics of an overpass bridge in a full-scale destructive test", Journal of Engineering Mechanics, 2013, 139 (6), pp. 691-701.

Sun, S., Zhang, C., Yu, G., "A Bayesian network approach to traffic flow forecasting", IEEE Transactions on Intelligent Transportation Systems, 2006, 7 (1), pp. 124-133.

Torfi, F., Farahani, R.Z., Rezapour, S., "Fuzzy AHP to determine the relative weights of evaluation criteria and Fuzzy TOP-SIS to rank the alternatives", Applied Soft Computing Journal, 2010, 10 (2), pp. 520-528.

Vagnoli, M., Remenyte-Prescott, R., Andrews, J., "Railway bridge structural health monitoring and fault detection: State-of-the-art methods and future challenges", Structural Health Monitoring, 2018, 17(4), 971-1007.

Vagnoli, M., Remenyte-Prescott, R., Andrews, J., "A fuzzy-based Bayesian Belief Network approach for railway bridge condition monitoring and fault detection", European Safety and Reliability Conference (ESREL) 2017, Portoroz, Slovenia, 18-22 June.

Wang, Y.-M., Elhag, T.M.S., "On the normalization of interval and fuzzy weights", Fuzzy Sets and Systems, 2006, 157 (18), pp. 2456-2471.

Zhao, X., Liu, H., Yu, Y., Xu, X., Hu, W., Li, M. and Ou, J., "Bridge displacement monitoring method based on laser projection-sensing technology", Sensors, 2015, 15(4), pp.8444-8463. 
Zheng, W., Yu, W., "Probabilistic approach to assessing scoured bridge performance and associated uncertainties based on vibration measurements", Journal of Bridge Engineering, 2015, 20 (6), art. no. 04014089. 